\title{
Para una historia social de la niñez rural. El caso patagónico. Algunos apuntes teóricos metodológicos
}

\author{
Enrique Mases
}

\section{Resumen}

Este artículo propone realizar una mirada sobre la construcción de la niñez en contextos de ruralidad y de desigualdad social, a partir de una perspectiva anclada en la historia social. Para ello acude a la delimitación territorial de la región rural de la Patagonia Argentina como estudio de caso, entre fines del siglo XIX y la primera mitad del siglo XX con el objeto de aportar a una historia de la marginalidad. El mismo, está planteado como un estudio crítico a partir del recorrido de una amplia bibliografía dedicada a esta temática y deja, a modo de sustancial aporte, algunos apuntes tanto teóricos como metodológicos para su abordaje.

Palabras clave: Niñez; Ruralidad; Desigualdad social; Patagonia.

For a social history of rural childhood. The Patagonian case. Some theoretical and methodological notes

\begin{abstract}
The intent of this article is to take a look at the construction of childhood in the contexts of the countryside and social inequality, from a perspective anchored in social history. To do this, it analyzes the territorial delimitation of the rural region of Argentine Patagonia, at the turn of the 20th century, as a case study in order to contribute to a history of marginalization. It is a critical study based on a wide bibliography dedicated to this subject and leaves some theoretical and methodological notes for its approach as a substantial contribution.
\end{abstract}

Key Words: Childhood; Countryside; Social inequality; Patagonia.

\footnotetext{
${ }^{(*)}$ Doctor en Historia por la Universidad de Buenos Aires. Especialista en Sociología de la Agricultura con orientación en Empleo y Reestructuración del Mercado de Trabajo Agrario (Universidad Nacional del Comahue). Profesor de Enseñanza Secundaria, Normal y Especial en Historia (UBA), Argentina. Mail: emases@ciudad.com.ar ORCID: https://orcid.org/0000-0002-3640-4117
} 
"Para una historia social de la niñez rural. El caso patagónico. Algunos apuntes teóricos metodológicos"

\section{Para una historia social de la niñez rural. El caso patagónico. Algunos apuntes teóricos metodológicos}

\section{Introducción}

La historia social como una arista de la disciplina histórica, la niñez rural como un actor social significativo y la desigualdad social como un fenómeno permanente en la sociedad capitalista son términos que han tenido un largo devenir en los procesos históricos. Lo novedoso tal vez sea la interrelación que los mismos han tenido en los últimos tiempos. En efecto, a partir de una nueva mirada de la historia social, que al incorporar en sus estudios el concepto de desigualdad, naturalmente ha debido ampliar la temática a abordar, incorporando entre otras, el análisis de la vida de estos pequeños actores del drama social, como son los niños de los sectores populares, que habitan esa ruralidad.

A partir de estas consideraciones previas, el presente trabajo intenta en la primera parte reflexionar acerca de la relación entre la historia social y la niñez particularmente la que mora en el espacio rural y la desigualdad, la que aparece como una condición central en la vida de estos verdaderos marginados en la historia argentina. En la segunda parte, desde del análisis de un espacio regional determinado - la Patagonia rural entre fines del siglo XIX y la primera mitad del siglo XX-pretendemos avanzar en una serie de reflexiones teórico metodológicas que ayuden a comprender las peculiaridades, particularidades, $\mathrm{y}$ al mismo tiempo conocer las dificultades de este tipo de estudios temáticos.

\section{La niñez y la historia social}

Olvidada y silenciada durante largos años, la historia social de la niñez ha sido una de las contribuciones disciplinares que mayor vigor y desarrollo ha tenido en los últimos tiempos a favor de una prolífica producción que incluyó distintas miradas, diferentes concepciones y hasta una diversidad metodológica, que arrojaron luz sobre el rico y diverso mundo de la niñez. En efecto, temas centrales como las prácticas pedagógicas, la cultura escolar y la vida cotidiana familiar, la judicialización de la infancia hasta las políticas estatales sobre la infancia y la emergencia de los discursos con una impronta basada en el psicoanálisis, son algunas de ellas (Carli, S. 2002; Zapiola, M., 2007; Cosse, I., Llobet, V., Villata, C., Zapiola, M., 2010; Lionetti y Miguez, 2010).

Sin embargo, este fuerte interés de los historiadores sobre la temática de la infancia, tiene todavía algunos espacios en blanco o pocos concurridos, particularmente los que tienen que ver con el trabajo infantil y la niñez en el mundo rural. En efecto, salvo algunos aportes pioneros 


\section{Enrique Mases}

como el de E. Ciafardo, J. Suriano y E. Pagani y M. Alcaraz a los que podríamos sumar más cercano en el tiempo las contribuciones de M. Averza y Y. de Paz Trueba (2014) el trabajo de los niños es un aspecto de la vida de estos poco desarrollado en términos de producción historiográfica. En un artículo publicado hace ya algunos años Juan Suriano (1990: 253) intentaba ensayar algunas respuestas al interrogante que planteaba este menor interés de los historiadores por el trabajo infantil señalando que:

\footnotetext{
"el niño trabajador ha sido y es un verdadero marginado de la historia, quizás por su inserción laboral sensiblemente inferior a la del adulto o por el carácter en general complementario y auxiliar del trabajo realizado, tal vez por su falta de protagonismo sindical o, simplemente, por la corta duración y la naturaleza transitoria del mismo. La invisibilidad propia del trabajo infantil (¿cómo inferir sobre las actividades de miles de niños que ayudaban a sus padres en las tareas domésticas?) o el carácter informal de la ocupación de un importante segmento de estos pequeños trabajadores debe de haber contribuido a desalentar a los investigadores."
}

Este mismo escenario, se repite, pero agravado, cuando revisamos lo escrito sobre el mundo de la niñez rural, ya que no solo no existen estudios generales que tengan como centro de atención la vida del niño rural, sino que para algunos escenarios regionales como el patagónico aparece aún hoy totalmente ignorado. Sin lugar a dudas, esto resulta paradójico ya que esta invisibilidad contrasta con el rol principal que el mundo rural tuvo en términos económicos, sociales y simbólicos durante buena parte de la historia moderna de la Argentina y de la Patagonia en particular. Por el contrario, la niñez rural se ha mantenido al margen de los trabajos científicos, especialmente desde un punto de vista histórico.

Algunas de las razones que invoca Suriano para intentar justificar el poco interés de los historiadores por el trabajo infantil pueden ser aplicados al niño rural incluyendo además que este sujeto histórico rara vez dejó un testimonio escrito salvo en algunas fuentes judiciales o en algún subregistro documental.

Sin embargo, ese niño tuvo una significativa centralidad en la vida de relación del ámbito rural a partir de encontrarse desde muy temprano en su existencia integrado al mundo del trabajo compartiendo saberes con los adultos e incluso asumiendo responsabilidades en las actividades productivas muy por encima de su nivel de madurez.

\section{Lo rural}


"Para una historia social de la niñez rural. El caso patagónico. Algunos apuntes teóricos metodológicos"

En la Argentina finisecular la mirada que se tenía sobre lo rural se asentaba sobre una serie de premisas que pasaban por entender que el mismo se definía a partir de un vasto espacio, con baja densidad de población, donde casi en forma excluyente predominaban las actividades agropecuarias y encerrado en sí mismo. Es decir que lo urbano y lo rural aparecían como compartimentos estancos con escasa capacidad de relacionarse entre sí por lo tanto también con escasa movilidad poblacional que pudiera transitar ambos espacios. Finalmente, esta mirada se construía desde lo urbano y desde allí se describía lo rural como un mundo separado de este. Como señalan Ortiz Bergia y Salomón (2017):

“el funcionamiento del espacio rural se basó en un enfoque dicotómico, en el que el campo era delimitado por su opuesto, la ciudad, según la variación de tamaño, formas y relación del hombre con la naturaleza. Las tradiciones sociológicas fueron importantes en la construcción de dicha conceptualización, al imprimir un fuerte acento binario a los sistemas de clasificación de la realidad, entre los que se destacó naturalmente la antinomia entre el campo y la ciudad".

Esta mirada que fue preponderante hasta los años sesenta sufrió algunas tensiones e impugnaciones cuando se puso en duda particularmente lo cerrado de uno y otro espacio y la nula interdependencia. Por el contrario, se empezó a visualizar la relación de los procesos que se desarrollaban en uno y otro ámbito en diferentes momentos, teniendo a los trabajadores como actores principales. Un trabajo pionero de Ofelia Pianetto (1984) sobre mercado de trabajo y acción sindical en la pampa húmeda no solo plantea la migración estacional de mano de obra entre campo y ciudad, sino que además demuestra que "La estrecha dependencia del mercado de trabajo agrícola - urbano determina que las variaciones que se presentan en el nivel ocupacional agrario se traduzcan en una situación similar en los centros urbanos de la pampa húmeda." Estas afirmaciones comenzaban a horadar aquella primera premisa de las características dicotómicas e inmutables de las esferas de lo urbano y lo rural.

Para el escenario patagónico esta doble circulación también se cumple para diversos actores urbanos y rurales. El traslado de los centros urbanos de familias con su proles, o de niños y niñas solas, para incorporarse en las distintas actividades estacionales que requieren una demanda significativa de mano de obra fue una constante en un amplio periodo que abarca las últimas décadas del siglo XIX y se extiende a buena parte del siglo XX, de la misma manera que ciertos actores rurales particularmente las niñas que, alcanzada cierta edad son extrañadas 


\section{Enrique Mases}

de su lugar de origen y colocadas como personal doméstico de familias que vive en pueblos cercanos o en ciudades más importantes.

A esta primera impugnación le siguió un segundo embate que, planteó frente a la explicación cuantitativa de lo rural, la posibilidad de ver a este como una construcción social en donde en este ámbito se desenvuelve un singular escenario con representaciones, matices y particularidades que lo hacen sumamente dinámico al contrario de la construcción anterior donde lo rural aparece como estático, cerrado y socialmente homogéneo. Sin lugar a dudas, los aportes de Raymond Williams (2001; p. 410) fueron significativos ya que este visualiza al campo no como una categoría sociológica, sino como un espacio cultural, construido por operaciones simbólicas que remiten a un imaginario social. De esta manera, atribuía importancia a lo cultural como un elemento esencial en la configuración y articulación de las relaciones sociales. Y aquí nuevamente lo sucedido con algunos ámbitos rurales patagónicos encuadran perfectamente en esta mirada de Williams.

En efecto, este es el caso de Chos Malal una localidad en el norte neuquino que más allá de lo cuantitativo comparte sus características culturales predominante en la zona rural neuquina. Capital del territorio y principal núcleo poblacional hasta 1904, se presenta ante las miradas de los viajeros con una pléyade de "habitantes que viven dedicados a las tareas de la vida de campo, no faltando el comercio representado por varias casas" (Carrasco, 1902: 74) A las preeminencias de las actividades rurales y de la población de origen campesino chileno, debemos agregar otros factores de análisis. La articulación económica de Chos Malal con el resto del territorio nacional no se diferencia de las características del campo neuquino, donde el aislamiento respecto del mercado nacional era suplido por la integración a los circuitos comerciales de la zona rural del sur de Chile. Es decir que, la similitud de los hábitos de consumo, vivienda, formas de sociabilidad y ritos públicos entre los habitantes de Chos Malal y los del resto del área rural, nos permiten pensar en una uniformidad cultural en toda la región, de lo que la antigua capital no estaba diferenciada.

\section{La desigualdad}

Del mismo modo que el tema de la niñez, la desigualdad fue una línea de investigación y reflexión significativa de la economía y las ciencias sociales, en modo especial de la sociología, pero no de la historia social por lo menos hasta los años noventa. En efecto, la desigualdad no fue abordada por los investigadores de la disciplina de manera directa y como un fenómeno en sí mismo, sino colateralmente a través de las diversas situaciones de explotación que estaban presentes en determinados procesos históricos. Es decir, se ocuparon de la cuestión, aunque a 
"Para una historia social de la niñez rural. El caso patagónico. Algunos apuntes teóricos metodológicos"

veces de una manera solapada dentro del marco de otros objetivos como el conflicto social y por el contrario no lo hicieron a través de enfoques en donde primaran los distintos tipos de desigualdades y sus causas. Esta forma de abordar la cuestión, impidió durante largos años, pensar la desigualdad en términos más complejos, como las inequidades de género, raza, etnia o de grupos sociales como el que nos ocupa. Como sostiene Juan Suriano (2017: 24) "como ha ocurrido a partir de los cambios económicos y sociales de los años ochenta, el amplio mundo de individuos que quedan fuera del mundo del trabajo formal y de las políticas de protección estatal".

En nuestro país, el desarrollo de la economía desde el advenimiento de las políticas neoliberales tuvo como consecuencia funesta la virulenta crisis económica y política cuya eclosión se produjo en diciembre de 2001. Ese proceso fue, para muchos historiadores, el disparador del interés por estudiar la desigualdad en la historia, la que veíamos reproducida al infinito durante la década de 1990, de forma incremental a medida que se acercaba el preanunciado fin del proceso (Santilli, D., 2019).

De esta manera en las últimas tres décadas el tema de la desigualdad ha crecido en visibilidad, mucho más a partir de la comprobación que la desigualdad y la pobreza no han disminuido, y, es más, es muy probable que esta situación no se revierta en el corto y mediano plazo a menos que los Estados impongan políticas que reviertan esta tendencia. En este marco, es que los historiadores han renovado sus preguntas a la historia para que las respuestas que esta da como ciencia se encuentren las claves para intentar aportar desde el propio métie a la construcción de una sociedad más justa.

Pero también, a la luz de estos nuevos acontecimientos es que la historia social debe recoger con mayor profundidad los desafíos que provocan las desigualdades contemporáneas en un país como la Argentina y en un espacio particular como es la Patagonia, que hoy resulta menos inclusivo que el de buena parte del siglo XX. En este marco es necesario un esfuerzo mayor para historiar socialmente el concepto de desigualdad y referenciarlo en procesos concretos que se sucedieron en el devenir de nuestra sociedad. Si las preguntas sobre el presente ayudan a interrogarnos sobre el pasado, el descubrimiento de estas desigualdades debe impulsarnos a los historiadores sociales a reconsiderar y profundizar algunos aspectos del pasado que hasta hace poco tiempo no merecían atención, entre ellos el tema de la niñez rural y las asimetrías existentes, respecto a las posibilidades de acceder a las condiciones de vida, de la que gozaba esa misma niñez en el mundo urbano patagónico o en otras regiones de mayor desarrollo del país.

\section{Algunas reflexiones teóricas}




\section{Enrique Mases}

Respecto al sujeto central de esta particular temática que es la niñez rural nos interesa plantear algunas consideraciones y definiciones sobre esta categoría histórica y los usos de la misma.

Una primera tiene que ver a que nos referimos cuando hablamos de niño y de la niñez. Según la mayoría de las normas jurídicas internacionales, niño es toda persona menor de 18 años. La mayor parte de los países del mundo han fijado asimismo la mayoría de edad civil en los 18 años. Amnistía Internacional utiliza esta definición, al igual que la mayoría de Organizaciones No Gubernamentales (ONG), y los grupos de defensa de los derechos del niño. En nuestro país, y en relación al artículo $1^{\circ}$ de la Convención sobre los derechos del niño: La República Argentina declara que el mismo debe interpretarse en el sentido que se entiende por niño todo ser humano desde el momento de su concepción y hasta los 18 años de edad. ${ }^{1}$

$\mathrm{Y}$ en este intento de definir lo que se entiende por niñez y teniendo en cuenta que la misma es una categoría histórica, social y cultural, nos pareció acertado las reflexiones de Juan Suriano, el cual ve a este concepto como una construcción sociocultural, que fue cambiando a lo largo del siglo. El mismo autor advierte que utiliza indistintamente el término infancia como sinónimos de niñez, adhiriendo a la forma en que entiende esta noción el Ministerio de Trabajo:

"La niñez es aquel periodo cronológico dentro del crecimiento de los seres humanos que comprende, según la Convención de los Derechos del Niño, desde el nacimiento hasta los dieciocho años de edad. La infancia hace referencia al estado evolutivo de un ser humano que abarca desde el nacimiento hasta los doce o catorce años aproximadamente. Por lo tanto, niñez, sustantivo colectivo de niños y niñas, incluye a la infancia, pero también a la adolescencia. Esta última es un periodo evolutivo particular de desarrollo que tiene características diferentes de la infancia y se extiende de los doce o catorce años de edad hasta los dieciocho aproximadamente" (Suriano, J., 2007).

Lo interesante de esta salida por la que opta este historiador es que evita el aumento de los equívocos en las definiciones, ya que introducir precisiones demasiado ajustadas a la edad biológica de los niños traería mayor confusión, necesitando aclaraciones constantes. Siendo, además, que la edad y la forma que ella es percibida, como categoría también es una construcción social que se ha modificado a lo largo del tiempo, de acuerdo a cada sociedad e incluso de las diferentes clases sociales al interior de la misma por lo que la extensión de la niñez no es parámetro universal y homogéneo.

${ }^{1}$ Congreso Nacional Ley N 23849 Convención de los Derechos del Niño. Promulgada 16 de octubre de 1990. 
"Para una historia social de la niñez rural. El caso patagónico. Algunos apuntes teóricos metodológicos"

A partir de estas definiciones, abordar la cuestión de la niñez, y teniendo en cuenta el periodo de estudio abarcado, se la puede definir, como aquél lapso de tiempo que va desde el nacimiento hasta la emancipación de ese niño o niña que incluye la infancia, pubertad y adolescencia de los mismos, sin un límite preciso en cuanto a la edad. La niñez, la infancia, y la adolescencia involucran interpretaciones laxas pudiendo variar la asignación de características y extensión de la vida humana, aún en una misma época, según el organismo, institución o disciplina de que se trate.

Es decir que este niño forma parte primero de una familia y luego de una comunidad por lo que la vida de este está estrechamente ligado a las pautas de crianza, pero también a los contextos socioeconómicos, y a los intereses sociopolíticos. Por lo tanto, no se puede indagar sobre el mundo de la niñez sin pensarlo en sus diferentes relaciones con el mundo de los adultos. Como sostiene Claudia Freidenraij (2020:45) "Dado que la infancia es una construcción histórica fuertemente atravesada por el poder, las infancias se desarrollan siempre en el marco de relaciones "contingentes" y "asimétricas". Se trata de vínculos asimétricos marcados por la verticalidad y la subordinación de parte de los niños respecto de los adultos".

De la misma manera y por las mismas razones es necesario considerar a la niñez en el marco de relaciones que se construyen en su entorno familiar. Como señala L. Stone (1990) es imposible estudiar a los niños al margen de sus padres, ya que la historia de la infancia es la historia de las formas en que los padres han tratado a sus hijos. Por lo tanto, indefectiblemente al llevar adelante esta investigación necesariamente debimos tener en cuenta a la familia, que es la institución donde interactúan no solo los niños sino también los padres.

Pero también el niño forma parte de una comunidad y de las representaciones sociales que tiene esa comunidad, por lo tanto estudiar la niñez rural patagónica significa un constante esfuerzo en visualizar las características particulares de aquellos niños que provienen de familias de inmigrantes de diversos orígenes raciales y culturales, de familias criollas o de comunidades indígenas que encierran diferentes sistema de tradiciones, valores y de aspiraciones y a los que solo los iguala paradójicamente el rasero de la desigualdad.

\section{Aspectos metodológicos}

En cuanto a los aspectos metodológicos debemos señalar que el universo de análisis, cuando se aborda el espacio patagónico necesariamente se debe realizar un recorte ya que son muy diferentes las condiciones materiales de vida de los niños de los sectores populares rurales, de los hijos de los propietarios de estancias o de importantes establecimientos agropecuarios y de aquellos infantes que junto a sus padres están radicados en los campamentos de los grandes 


\section{Enrique Mases}

emprendimientos estatales como las explotaciones petrolíferas o las obras hidráulicas entre otros. Como señalamos más arriba la razón de esta segregación está dada porque esos niños tienen una mejor calidad de vida, caracterizada por una forma diferente de acceso a la educación, a la salud y otros bienes materiales que los diferencian de los otros niños que pueblan el mundo rural.

Respecto al acceso a la educación, como señala M. Pierini (2009: 226) los estancieros contrataban docentes o personas idóneas para que impartieran a los niños en sus hogares los conocimientos básicos debiendo luego continuar sus estudios en internados en otras localidades, tanto en Santa Cruz como en Buenos Aires o Europa. De la misma manera las condiciones de vida de los niños en los emprendimientos estatales con la posibilidad de acceso concreto a la educación ingresando en los cursos regulares de la escuela pública, a la salud a través de la medicina científica y los niveles de consumo existentes en los diferentes campamentos estatales los alejan definitivamente de los otros pequeños actores del mundo rural (Mases, E, y Otros, 1994).

Otro aspecto metodológico a considerar es el que tiene que ver con la noción de trabajo infantil que es aquella que comprende los múltiples modos y formas que adquiere, tanto el trabajo remunerado (formal, informal, estable e inestable), como el no remunerado (doméstico y de cuidado). En efecto, necesariamente cuando nos referimos al trabajo de los niños en el ámbito rural, debemos prestar especial atención a una serie de consideraciones o advertencias previas, que nos permiten abordar con cierta justeza un escenario laboral multiforme y ampliamente heterogéneo. Precisamente estas características nos obligan a diferenciar en primer lugar entre un trabajo de tipo doméstico o en el seno de una economía familiar, de aquel donde el infante o adolescente vende real o figuradamente su fuerza de trabajo; diferenciándose estos dos a su vez de un tercero que tiene que ver con aquel comprendido en instituciones estatales o religiosas y de un cuarto que son los niños incluidos en los repartos judiciales.

Una segunda advertencia tiene que ver con el trabajo de la niñez en el seno de la economía familiar donde no siempre son solo los hijos los que realizan las distintas labores. En efecto, dentro del trabajo infantil familiar una forma bastante difundida es la actividad que llevan adelante niños que no forman parte de la familia biológica pero que, si la integran circunstancialmente, que su incorporación a la misma deviene de diferentes situaciones y tienen distintas acepciones. Estos son los denominados agregados, entenados, los prestados y finalmente los criados.

Cada una de estas modalidades, que hemos señalados hasta aquí, implica condiciones y relaciones diferentes entre el propio actor y el mundo laboral en el cual está inmerso, pero 
"Para una historia social de la niñez rural. El caso patagónico. Algunos apuntes teóricos metodológicos"

también la percepción de los adultos respecto a la actividad que realizan estos peculiares trabajadores es totalmente distinta dependiendo de la situación.

Siguiendo con los aspectos metodológicos, es preciso señalar que respecto al mundo de la niñez rural podemos -y de hecho lo hacemos-, utilizar indistintamente las denominaciones de niño o infante y en algunas ocasiones la de púber o joven pero no la de menor ya que esta es una categoría diferente que tiene limitado su uso a las cuestiones legales y penales y por lo tanto la utilización solo es posible en aquellas situaciones puntuales donde el niño aparece relacionado con el mundo de la justicia. Efectivamente, donde aparece por primera vez este término es en la ley de Patronato de Menores o comúnmente denominada Ley Agote (10.903) y la misma se estructura en torno a la categoría menor, que designa a todo niño y adolescente delincuente y/o material o moralmente abandonado (Zapiola, M., 2006: 69).

Esta aparece como una categoría laxa y confusa en la que se englobaban a huérfanos, abandonados, delincuentes, viciosos y vagos. Es decir, todos los menores de edad tutelados por el Estado. Por lo tanto, esta categoría hacía referencia, en un principio, a un conjunto urbano muy heterogéneo, pero uniformemente ajeno a las pautas de comportamiento, circulación por el espacio, educación, trabajo, sexualidad y socialización familiar y extra familiar que las elites encontraban para los menores de edad.

Dicho de otra manera, los menores eran niños expuestos a la condición de pobreza, desnutrición, ausencia de protección, escolarización y futuro; pero esas mismas carencias conllevaban la peligrosidad en tanto podían inducir hábitos contrarios a las buenas costumbres y perjudiciales a los valores con lo que se envestía la moderna concepción de la infancia. La imagen prototípica del menor eran los huérfanos, aunque muchas veces con padres vivos, abandonados en instituciones de beneficencia por diversas razones, entre las que no estuvo ausente la intención de sus progenitores de salvaguardarlos de su condición de pobreza (Lionetti, L. y Miguez, D., 2010: 21).

En lo que respecta al ámbito rural patagónico el tema de los menores en emergencia fue un área de intervención de dos instituciones particularmente. Una tiene que ver con sociedades benéficas tanto de la sociedad civil pero particularmente de la Iglesia Católica y la otra es la justicia a través de funcionarios como los Defensores de Menores que colocaban a estos menores en familias sustitutas, especialmente a los niños indígenas que eran arrebatados a sus progenitores en el marco de la desestructuración de la antigua organización parental de las comunidades indígenas.

Otra de las dificultades metodológicas que debemos advertir es que, a causa de su ilegalidad, de la escasez de fuentes escritas, de los problemas para su registro y los pocos análisis que abordan esta temática en el plano historiográfico hace que el abordaje de algunos aspectos de la niñez, 


\section{Enrique Mases}

particularmente el que tiene que ver con el trabajo infantil presente una serie de inconvenientes que es preciso señalar.

Una primera y principal es la falta de fuentes cuantitativas. No hay registros ni estadísticas públicas y privadas que marquen fehacientemente la presencia de la niñez trabajadora a partir de consignar la edad de los trabajadores. Tampoco existe una uniformidad de criterios en los datos suministrados tanto por los censos territorianos como nacionales ya que no hay un criterio único en la forma de registrar el trabajo de aquellos que transitan la niñez y la pubertad. En realidad, salvo el censo nacional de 1914 que registra trabajo infantil industrial y comercial en el resto no aparecen datos acerca de la actividad laboral de estos pequeños trabajadores.

Esto que es válido para el plano nacional también se repite en la Patagonia con una excepción que vale la pena remarcar y es el que tiene que ver con el censo nacional de 1895 de Tierra del Fuego. En el mismo, tal vez por el escaso número de habitantes -solo 447 personas - el total de los censados están registrados con indicación de edad origen y profesión. Este criterio censal nos permite advertir la presencia de niños que a pesar de su corta edad ya figuran con una profesión u oficio.

En Ushuaia: Sagastume Ramón (13años) jornalero; Zelaya, Martín (14 años) carpintero; Leitano, Francisco (12 años) carpintero; Mazza Francisco (17 años) carpintero; Ingoloti, Francisco (12 años) herrero; Cintani, Livio (13 años) herrero; Sanchez, Francisco (16 años) carpintero. En Haberton: Bridges, Samuel (17 años) estanciero; Shucaiagu, Julia (11años) sirvienta; Galarium, Edward (11 años) peón. En la Isla de los Estados: Limares, Eduardo (16 años) marinero. ${ }^{2}$

Pero como señalamos precedentemente el caso del territorio fueguino es casi una excepción que está acompañado en esa excepcionalidad por otras fuentes dispersas y fragmentadas como son algunos censos policiales llevados a cabo en el territorio de La Pampa. Por lo tanto, el trabajo infantil aparece invisibilizado en las estadísticas y por ende también en los repositorios oficiales. Estos problemas estadísticos también las encontramos cuando abordamos el tema de la salud en los territorios patagónicos donde aparecen claramente las dificultades que tuvieron las autoridades nacionales para la conformación de una estadística sanitaria las que aparecen parciales y fragmentadas. Así en los primeros años de este estudio las series que están más completa son las que tienen que ver con las campañas de vacunación antivariólica.

En cambio, de otras "epidemias (tifus, fiebre amarilla, peste bubónica, cólera) o de enfermedades sociales, como la tuberculosis y la sífilis, los facultativos daban fe de su existencia, pero en muchos casos, con un registro más impresionista que estadístico" (Di Liscia, M., 2009: 264) Recién en los comienzos de la década del cuarenta, aparece un primer registro

${ }^{2}$ Fuente: Censo Nacional de 1895 de Tierra del Fuego. Citado por Belza, Juan E. En la isla del fuego $2^{\circ}$ Colonización. Buenos Aires, Instituto de Investigaciones Históricas Tierra del Fuego, 1975. pag. 41-48. 
"Para una historia social de la niñez rural. El caso patagónico. Algunos apuntes teóricos metodológicos"

institucional referido a recursos humanos e infraestructura existente en los territorios nacionales incluidos los patagónicos.

Junto al problema de las estadísticas debemos sumar en lo cuantitativo algunas dificultades con los censos nacionales debido a los diferentes criterios censales que se adoptaron en cada uno de ellos particularmente el confeccionado en 1947 que difiere de los dos anteriores de 1895 y 1914 en algunos ítems. Estas diferencias pasan porque en el confeccionado durante administración peronista junto a los territorios nacionales en que estaba dividida políticamente la Patagonia se le suma la gobernación militar de Comodoro Rivadavia conformada con parte del territorio de Chubut.

La segunda tiene que ver con considerar solo población urbana aquellos núcleos de población con más de 2000 habitantes con lo que en el Territorio Nacional de Tierra del Fuego no figura con población urbana a diferencia de los censos anteriores. Finalmente, una tercera dificultad se da en la distinta clasificación de la población infanto juvenil de acuerdo a su edad ya que mientras los primeros censos nacionales abarcan desde el nacimiento hasta los 17 años agrupados en tres grupos en cambio el censo nacional de 1947 las subdivide en cuatro grupos que van del nacimiento a los 19 años de edad por lo que resulta imposible la comparación ya que el último cuartil incluye personas que están fuera de la definición de niño aquí adoptado.

Una segunda dificultad tiene que ver con la escasez de fuentes cualitativas particularmente de repositorios oficiales por lo que entonces la búsqueda debió centrarse en repositorios alternativos como lo son los archivos judiciales los que si nos brindan cierta información, a partir de aquellos expedientes que se refieren a distintos tipos de accidentes fundamentalmente aquellos relacionados con el trabajo infantil, o los que tienen que ver con la Defensoría de Menores o causas penales donde están involucrados estos niños. Pero además éstos mismos expedientes contienen una valiosa información, la que, a través del testimonio de la víctima, su entorno familiar y los testigos ocasionales, incluso el o los victimarios, nos describen el escenario socioeconómico donde se desenvuelven la vida de estos pequeños actores.

Junto a los archivos judiciales otra fuente que resulta de suma utilidad son las Guías de las Escuelas confeccionadas a partir de directivas del Consejo de Educación. Las mismas consistían en una concisa descripción que los maestros de los Territorios debían llenar y remitir al Consejo en las que se consignaban datos tales como: ubicación y modo de arribo al paraje desde la estación Constitución, datos sobre las condiciones edilicias, características de la población escolar como, nacionalidad, modo de vida, origen, salud, vivienda, y medios materiales de vida, origen, salud, vivienda, y medios materiales de existencia de la familia. Como se puede inferir, estas guías constituyeron en su momento un documento útil para poner en conocimiento a los postulantes del lugar de trabajo para el futuro docente, pero cobran hoy mayor significatividad 


\section{Enrique Mases}

por cuanto constituyen un documento cuantitativo y cualitativo de las condiciones de vida de la totalidad de los territorios.

Finalmente digamos que las entrevistas personales sumados a testimonios insertos en memorias, ensayos, autobiografías y en la prensa. Respecto de las primeras debemos señalar que en la investigación podemos utilizar como fuentes dos tipos de entrevistas de acuerdo a su origen: por un lado, aquellas llevadas a cabo por nosotros mismos, es decir fuentes primarias; y por el otro también aquellas que han llevado adelante otros investigadores, las que nos resultan de suma utilidad, en muchos casos para ejemplificar nuestras argumentaciones.

\section{Algunas reflexiones finales}

Acometer el estudio de una historia social de la niñez rural, en este caso en el escenario patagónico, no es una tarea sencilla, por el contrario, es recorrer un camino lleno de obstáculos y dificultades metodológicas de todo tipo como ya lo hemos descripto someramente. Sin embargo, reconstruir su vida material el acceso a la salud y a la educación, su sistema de representaciones, sus angustias y alegrías, su tiempo libre, en definitiva, su dura y peligrosa vida en sociedad, sigue siendo un desafío atractivo, ya que el mismo implica rescatar del olvido a este pequeño actor que sin lugar a dudas cumplió un papel central, aunque silencioso y subordinado, en una sociedad rural de frontera en un tiempo determinado. Es decir, resulta estimulante y a la vez esclarecedor reconstruir la vida de estos niños que habitan esa ruralidad, los que transitan, inestables e inseguros, una niñez efímera, esforzada y escasamente reivindicada.

\section{Bibliografía}

Carli, S. (2002). Niñez, pedagogía y política. Transformaciones de los discursos acerca de la infancia en la historia de la educación argentina entre 1880 y 1955. Buenos Aires: Miño y Dávila, p. 19-25.

Carrasco, G. (1902). De Buenos Aires a Neuquén. Reseña geográfica - industrialadministrativa. Buenos Aires, s.n.e., p. 74.

Cosse, I. (2010). Desconciertos frente al nuevo modelo de la crianza. Madres y padres en la Argentina de 1960. En L. Lionetti y D. Míguez: Las infancias en la historia argentina. Intersecciones entre prácticas, discursos e instituciones (1890-1960). Rosario: Prohistoria, p. 237-256. 
"Para una historia social de la niñez rural. El caso patagónico. Algunos apuntes teóricos metodológicos"

Cosse, I. Llobet, V. Villalta, C. y Zapiola, C. (eds.) (2011). “Introducción” a Infancias: política y saberes en Argentina y Brasil. Siglos XIX y XX. Buenos Aires: Teseo, pp. 11-28.

Di Liscia, M. (2009). Cifras y problemas. Las estadísticas y la salud en los Territorios Nacionales (1880-1940. En: Revista Salud Colectiva. Universidad Nacional de Lanús. Vol. 5 Nº 2, p. 264.

Freidemraij, C. (2020). La niñez desviada. La tutela estatal de niños pobres, huérfanos y delincuentes. Buenos Aires 1890-1919. Buenos Aires: Biblos, p. 45.

Lionetti. L y Míguez. D. (2010). Aproximaciones iniciales a la infancia en L. Lionetti y D. Míguez: Las infancias en la historia argentina. Intersecciones entre prácticas, discursos e instituciones (1890-1960). Rosario: Prohistoria, pp. 9-32; 24.

Mases, E, y Otros. (1994) El mundo del trabajo: Neuquén 1884-1930. Neuquén, GEHiSo, pp. 151-152.

Ortiz, B. y Salomón, C. (2017). Estado y bienestar rural argentino en la primera mitad del siglo XX. Un problema historiográfico. En: Historia Caribe - Volumen XII Nº 31 - Julio-Diciembre pp 19-44.

Paz Trueba de Y. (2014). El trabajo infantil en el centro y sur de la provincia de Buenos Aires. Niños y niñas a fines del siglo diecinueve y principios del veinte. En Revista Mundos do Trabalho. vol. 6 n. 12. p. 177-195.

Pianetto, O. (1984). Mercado de trabajo y Acción Sindical en la Argentina. 1890-1922. En: Desarrollo Económico. Buenos Aires. V 24. №94, julio-septiembre.

Pierini, M. (2009). Iglesia y Estado en los comienzos de la educación en Santa Cruz: Los salesianos. En: OSSANA, Edgardo (director). La educación en la Patagonia Austral: el rol del Estado. Historia de la educación de la Patagonia Austral. Tomo III. Rosario: Prohistoria Ediciones. Universidad Nacional de la Patagonia Austral, p.226.

Santilli, D. (2019). Dos siglos de desigualdad. ¿Qué sabemos? Un parcial estado del arte en el siglo XIX desde la historia. En: Quinto Sol. Vol. N23, N², 2019. Pp. 18-19.

Stone, L. (1990). Familia, sexo y matrimonio en Inglaterra, 1500 1800. México: F.C.E.

Suriano, J. (1990). Niños trabajadores. Una aproximación al trabajo infantil en la industria porteña a comienzos de siglo. En D. Armus (comp.). Mundo urbano y cultura popular. Estudios de Historia Social Argentina. Buenos Aires: Sudamericana, 1990. p. 253.

Suriano, J. (2007). El trabajo infantil. En: S. Torrado (comp.). Población y bienestar en la Argentina del primero al segundo Centenario. Una historia social del siglo XX. Buenos Aires: Edhasa, Tomo II, p. 335-336. 


\section{Enrique Mases}

Suriano, J. (2017). Problemas e interrogantes de la historia social en torno a la desigualdad. En: Revista Electrónica de Fuentes y Archivos. Centros de Estudios Históricos "Prof. Carlos A. Segretti”. Córdoba (Argentina), año 8, número 8, p. 24.

Williams, R. (2001). El campo y la ciudad. Buenos Aires: Paidós, p.410.

Zapiola, María C. (2006). ¿Es realmente una colonia? ¿Es una escuela? ¿Qué es? Debates parlamentarios sobre la creación de instituciones para menores en la Argentina 1875-1890. En: D. Lvovich y J. Suriano. Las políticas sociales en perspectiva histórica. Argentina, 1870-1952. Buenos Aires: Prometeo libros, p.69.

Suriano, J. (2007). La invención del menor. Representaciones, discursos y politicas públicas de menores en la ciudad de Buenos Aires, 1882-1921, Tesis de Maestría, Buenos Aires, UNSAM.

\section{Fuentes}

Censo Nacional de 1895 de Tierra del Fuego. Citado por Belza, Juan E. En la isla del fuego $2^{\circ}$ Colonización. Buenos Aires, Instituto de Investigaciones Históricas Tierra del Fuego, 1975. pag. 41-48.

Congreso Nacional Ley No 23849 Convención de los Derechos del Niño. Promulgada 16 de octubre de 1990.

Recepción: 20/10/2021

Evaluado: 17/11/2021

Versión Final: 14/12/2021 